\title{
A Focus on the Diagnosis of Early Rheumatoid Arthritis
}

\author{
Marta Olivieri*, Maria Chiara Gerardi, Francesca Romana Spinelli, Manuela Di Franco \\ Rheumatology Unit, Department of Internal Medicine and Medical Specialities, Sapienza University of Rome, Rome, Italy. \\ Email: *marta.olivieri11@gmail.com
}

Received September $20^{\text {th }}, 2012$; revised October $30^{\text {th }}, 2012$; accepted November $15^{\text {th }}, 2012$

\begin{abstract}
Nowadays it is worldwide accepted that early diagnosis and early treatment of Rheumatoid Arthritis (RA) can improve the prognosis in most of patients. In this way, the 2010 ACR/EULAR Rheumatoid Arthritis classification criteria have shown to be more sensitive than the ACR 1987 criteria and include better patients with early RA. Other important point to focus on is to identify predictive factors for outcome, in order to propose a more aggressive treatment for early RA patients who could develop a persistent and/or erosive disease. The presence of Rheumatoid Factors (RF) and Anticitrullinated peptides antibobies (ACPA), as well as the duration of the disease at the time of diagnosis, are independent risk factors for the development of erosive RA. As for imaging, both traditional X-ray and Magnetic Resonance Imaging (MRI) highlight respectively the Rapid Radiological Progression (RRP) and the presence of bone edema which are associated to a more aggressive disease. In the last years, the musculoskeletal ultrasonography (MSUS) has emerged as a useful imaging technique since it allows to identify synovitis and bone alteration earlier than the radiological examination. Interating clinical, serological and imaging data the clinician can define the effective disease activity of each patient.
\end{abstract}

Keywords: Early Rheumatoid Arthritis; Diagnosis

\section{Introduction}

Rheumatoid arthritis (RA) is a chronic systemic inflammatory disease affecting $1 \%$ of the population. It is associated with significant morbidity, mortality, and high burden for the National Health Service (NHS). The disease is characterized by inflammation of the synovium, most frequently occurring in the small joints of the hands and feet, but also large joints can be involved; the inflammatory process frequently leads to loss of cartilage and bone erosions. The joint destruction is correlated with the severity of inflammation [1]. If patients are no treated, work disability, comorbidity and mortality can generally occur within ten years. In the last years, development of new biological drugs directed against molecular targets known as key pathogenetic factors (cytokynes such as TNF alfa, interleukin-6), has improved the prognosis of the disease with significant reduction of radiological erosive damage and disability.

\section{Classification Criteria}

In the recent past, 1987 Classificative Criteria of American College of Rheumatology (ACR) have been used for the diagnosis of RA [2]; however, these criteria are un-

*Corresponding author. able to identify the disease at its onset [3]. Moreover, these criteria do not include laboratory findings, such as anti-citrullinated peptide antibodies (ACPA) that are more specific for RA than Rheumatoid Factor (RF). New classification criteria has been validated in 2010 by ACR and European League Against Rheumatism (EULAR) [4] and include 4 items: joint involvement (number of joints with definite synovitis), presence and titre of RF and ACPA, evaluation of acute-phase reactants such as Creactive protein (CRP) and erithrocyte sedimentation rate (ESR), and duration of symptoms (more or less than 6 weeks). Patients can be classified as RA if a score of $\geq 6$ out of 10 is obtained. Thus, RA can be diagnosed using a combination of clinical and serological parameters.

Even if they are classificative and not diagnostic, ACR 2010 criteria and 1987 criteria have different specificity and sensitivity to identify early RA patients [3]. Indeed some studies have compared the specificity and sensitivity of both classification criteria showed better sensitivity in 2010 criteria $(73.5 \%)$, and a better specificity in those of 1987 (92.9\%) [5].

Better performance of the 2010 criteria in term of sensitivity but not of specificity has been confirmed in a recent work carried out on polyarthritis patients with less than 6 weeks disease duration: in this cohort, only $71.5 \%$ of patients with a score of $\geq 6$ points at the first 
evaluation has been later classified as having RA [6].

\section{Pathogenesis and Antibodies}

The pathogenesis of RA implies an interaction between environmental factors and genetic background: in genetically predisposed individuals, an arthritogenic peptide may trigger the innate immune response which is followed by an upregulation of adaptive immunity. The earliest, preclinical phase of the disease, in which asymptomatic subjects develop ACPA and IgM-RF has been clearly demonstrated in recent studies on healthy blood donors who later have developed RA [7]. RF are antibodies (IgM, IgG, IgA) directed against $\mathrm{Fc}$ region of $\mathrm{IgG}$; RF (usually IgM) is not specific for RA, as it is also detected in other connective tissue diseases, infections, and autoimmune disorders, as well as in $1 \%-5 \%$ of healthy people. The presence of RF represent an independent risk factor for bone erosion and seems to predict radiographic progression [8]. The autoantibodies directed against citrullinated cyclic pepetides belong to a group of antibodies able to react with several citrullinated peptides on multiple proteins (filaggrin, vimentin, fibrin, alpha-enolase), and for this reason, indicated as ACPA [9]. These autoantibodies are found in $70 \%$ of RA patients and are highly specific; APCA also represent early predictors identifying patients at risk for more aggressive and erosive disease [10].

\section{Early Diagnosis}

Early diagnosis, as early as the symptoms of disease begin, is mandatory since early treatment is known to improve prognosis in most of patients. It is necessary to identify as soon as possible those subjects who might develop an aggressive disease and carry out all possible strategies to slow the disease progression [11]. Most important questions in the last years are: when does RA start? Can it be stopped [11]? In the preclinical phase, the adaptive immune response impairs the synovial membrane, leading to an influx of immune and inflammatory cells and increased vascularity of the joints followed by a destructive synovitis. In the asymptomatic phase, it is not feasible to identify the beginning of the disease from a biological point of view. An early diagnosis can be made as soon as the first clinical symptoms of arthritis start. The concept of a "window-of-opportunity" suggests that there is a period, early in the course of the disease, between biological changes and clinical evidence. In this period the disease process can be altered or maybe even reversed with a complete return to normality. Therapy during this period may have a much greater effect than treatment at a later stage in terms of halting disease progression and achieving remission [12]. "Early arthritis" is defined as symptomatic arthritis appeared from less than 24 weeks and "very early arthritis" from no more than 12 weeks. This range of time results from the evidence that $70 \%$ of patients reveal an irreversible joint damage after 2 years of disease, but $28 \%$ of RA patients already shows erosions at disease onset [13]. On the other hand, though the diagnosis of RA should be made as soon as possible, the differential diagnosis, particularly at an early stage, can be difficult and should include other arthritis such as spondyloarthritis, reactive arthritis, connective tissue disease and microcrystalline arthritis. As clinical evidence strongly supports the observations that structural damage occurs early and permanently in RA and intervention slows the progression of damage, a rapid referral to a rheumatologist when RA is suspected is recommended. In order to identify early disease, referral recommendation has been developed to help primary care physicians. Patients with any of the following criteria should be referred to the rheumatologist [14]:

- $\geq 3$ swollen joints;

- metatarsophalangeal/metacarpophalangeal involvement;

- morning stiffness $\geq 30 \mathrm{~min}$.

If the first key point is an early diagnosis, the second one is to identify the predictive factors of disease outcomes [15]. In the last years, different predictive models have been developed to identify in a very early stage of inflammatory arthritis those patients that will develop a persistent and/or erosive RA [16] and will need a more aggressive treatment.

In these models the presence of ACPA and the disease duration at the time of diagnosis have been identified as independent risk factors for the development of erosive RA and of persistent synovitis; particularly, the ACPA positivity is significantly linked to RA diagnosis [17].

As recommended by the ACR, a periodic measurement of disease activity with validated indices to evaluate disease activity and response to the therapy is mandatory [18]. The composite disease activity indices such as DAS28 (Disease Activity Score 28 joints), SDAI (Simplified Disease Activity Index 28 joints) and CDAI (Clinical Disease Activity Index 28 joints), allow the rheumatologist to define the arthritis activity discriminating between low, moderate and high disease activity [19] (Tables 1 and 2).

The earlier inflammatory arthritis is detected and treated, better the long-term outcome in terms of damage and function is obtained. Further benefit is shown with regular monitoring of disease activity and rapid therapeutic escalation to gain disease control. Sensitive measures are therefore required for the assessment and monitoring of treatment in early arthritis.

As for imaging, the $\mathrm{X}$-ray examination is still the first choice in the evaluation of erosive damage; however, even though it is highly specific, its sensitivity is low.

Patients that showed bone erosions in early stage of 
Table 1. Clinimetric index in RA.

\begin{tabular}{|c|c|c|c|}
\hline Elements & SDAI & CDAI & DAS28 \\
\hline Number of swollen joints & Simple count $(0-28)$ & Simple count $(0-28)$ & Simple count, square root Transformed \\
\hline Number of tender joints & Simple count $(0-28)$ & Simple count $(0-28)$ & Simple count, square root Transformed \\
\hline Acute phase reactants & $\mathrm{CRP}$ in $\mathrm{mg} / \mathrm{dL}(0.1-10.0)$ & - & ESR, log transformed \\
\hline Patient global health & - & - & VAS in $\mathrm{mm}$ \\
\hline Patient global disease activity & VAS in $\mathrm{cm}(0-10.0)$ & VAS in $\mathrm{cm}(0-10.0)$ & - \\
\hline Evaluator global disease activity & VAS in $\mathrm{cm}(0-10.0)$ & VAS in $\mathrm{cm}(0-10.0)$ & - \\
\hline Total index & $\begin{array}{l}\text { No immediate scoring due to } \\
\text { CRP; simple possible }\end{array}$ & $\begin{array}{l}\text { Immediate scoring possible; } \\
\text { simple calculation possible }\end{array}$ & $\begin{array}{l}\text { No immediate scoring due to ESR; } \\
\text { calculator required }\end{array}$ \\
\hline
\end{tabular}

Legend: CRP (creactive protein), ESR (erithrocyte sedimentation rate), VAS (visual analogue scale), DAS28 (disease activity score 28 joints), SDAI (simplified disease activity index 28 joints), and CDAI (clinical disease activity index 28 joints)

Table 2. Instruments to measure rheumatoid arthritis disease activity and to define remission.

\begin{tabular}{|c|c|}
\hline Instruments & $\begin{array}{c}\text { Thresholds of disease } \\
\text { activity levels }\end{array}$ \\
\hline $\begin{array}{l}\text { Disease activity score in } 28 \text { joints } \\
\text { (DAS 28) (range } 0 \text { - 9.4) }\end{array}$ & $\begin{array}{l}\text { Remission: }<2.6 \\
\text { Low activity: } \geq 2.6 \text { to }<3.2 \\
\text { Moderate activity: } \geq 3.2 \text { to } \leq 5.1 \\
\text { High activity: }>5.1\end{array}$ \\
\hline $\begin{array}{l}\text { Clinical disease activity index } \\
\text { (CDAI) (range } 0 \text { - 76.0) }\end{array}$ & $\begin{array}{l}\text { Remission: } \leq 2.8 \\
\text { Low activity: }>2.8 \text { to } 10.0 \\
\text { Moderate activity: }>10.0 \text { to } 22.0 \\
\text { High activity: }>22\end{array}$ \\
\hline $\begin{array}{l}\text { Simplified disease activity index } \\
\text { (SDAI) (range } 0-86.0 \text { ) }\end{array}$ & $\begin{array}{l}\text { Remission: } \leq 3.3 \\
\text { Low activity: }>3.3 \text { to } \leq 11.0 \\
\text { Moderate activity: }>11.0 \text { to } \leq 26 \\
\text { High activity: }>26\end{array}$ \\
\hline
\end{tabular}

disease detected by X-ray (Rapid Radiological Progression-PRP) have a worst outcome and need of early and aggressive treatment.

It has been demonstrated that RRP, defined as an increase of 5 or more points in radiological scores such as Sharp/van der Heijde score (SHS) in the first year of treatment, is associated with worst functional ability in later and more radiological damage progression in long term follow-up. Thus, RRP in the first year of treatment is an independent predictor of later functional disability, and represents not only a radiologically but also a clinically relevant early outcome helpful to define the initial choice of treatment [20].

This may mean that, as earlier studies have shown, patients with a low risk of RRP require less intensive initial therapy to prevent radiological damage progression than patients with a high risk [21,22].

Magneting Resonance Imaging (MRI) is shown to perform better than conventional radiology to identify articular alterations in RA patients, particularly bone edema, precursor of erosions, as early as after 4 weeks from the onset of symptoms [23] and gives $65 \%$ of sensitivity and $82.5 \%$ of specificity [24].

Muscoloskeletal ultrasound (MSUS) is cheaper and more accessible even if it is dependent on examiner and the reproducibility should be improved. It allows to identify the presence of synovitis, structural abnormalities of tendons, ligaments, entheses and bone alteration such as erosions before they can be detected by conventional X-ray examination [25]. Moreover through power Doppler (PD) it is possible to visualize the vascularity of the synovium that is linked to the presence of an active synovitis [26, 27]. It has been recently shown a bigger sensitivity of MSUS compared with clinical examination in identifying articular inflammation in "early undifferentiated arthritis" (earlyUA) patients with evidence of sub-clinical disease in $64 \%$ of patients [28]. Although further studies are required to assess the value of MRI and MSUS in the differential diagnosis of early arthritis, the findings of erosions and synovitis are useful in clinical practice to confirm suspected cases of RA. Furthermore, the detection of erosions and synovitis/PD signal on MSUS have a prognostic value and may be used to guide the choice of treatment strategies. Whilst MRI has been used as a monitoring tool in early arthritis, further research is ongoing for the standardisation of US as an outcome measure in response to treatment.

The importance of early diagnosis in RA patients is linked to the evidence of a better prognosis in patients treated in the early stage of the disease.

It has also been demonstrated that steroids therapy, started within the first 12 weeks shows clinical remission in a significant number of patients within 6 months [29] and that the therapy with Disease modifying anti-rheumatic drugs (DMARDs) is efficacy in $50 \%$ of patients if started within 12 weeks compared with $15 \%$ of patients if started after 20 weeks from the beginning of disease [30].

A recent meta-analysis carried out in 1133 RA patients with disease duration of less than 2 years, has shown that 
an average delay of 9 months in the treatment with DMARDs is associated with an increase of radiological progression [31].

\section{Conclusions}

As highlighted in this review, a key point of RA diagnosis is today represented by an early detection of patients at risk of a severe disease progression. Due to the complexity of disease, only the contemporary assessment of clinical features, laboratory tests and imaging findings can suggest the prognosis of each patient and drive the therapeutic approach. Early referral to rheumatologist is required when physicians suspect an inflammatory arthritis. Nowadays we have a diagnostic tool to diagnose early RA, even if it is can be improved.

The risk stratification allows to select patients who may need a more aggressive therapy since the earlier stage of the disease. Aim of the targeted therapy is to achieve a complete remission or, at least, a low disease activity as defined by clinimetric indices, early in the course of the disease and to warrant a clinical improvement at short and long term and a better quality of life.

\section{REFERENCES}

[1] M. C. Wick, S. Lindblad, L. Klareskog and R. F. van Vollenhoven, "Relationship between Inflammation and Joint Destruction in Early Rheumatoid Arthritis: A Mathematical Description," Annals of the Rheumatic Diseases, Vol. 63, No. 7, 2004, pp. 848-852. doi:10.1136/ard.2003.015172

[2] A. J. MacGregor, "Classification Criteria for Rheumatoid Arthritis," Bailliere's Clinical Rheumatology, Vol. 9, No. 2, 1995, pp. 287-304. doi:10.1016/S0950-3579(05)80191-8

[3] M. P. van der Linden, R. Knevel, T. W. Huizinga and A. H. M. van der Helm-van, "Classification of Rheumatoid Arthritis: Comparison of the 1987 American College of Rheumatology Criteria and the 2010 American College of Rheumatology/European League against Rheumatism Criteria," Arthritis \& Rheumatism, Vol. 63, No. 1, 2011, pp. 37-42. doi:10.1002/art.30100

[4] D. Aletaha, T. Neogi, A. J. Silman, et al., "2010 Rheumatoid Arthritis Classification Criteria: An American College of Rheumatology/European League against Rheumatism Collaborative Initiative," Annals of the Rheumatic Diseases, Vol. 69, No. 9, 2010, pp. 1580-1588. doi:10.1136/ard.2010.138461

[5] Y. Kaneko, M. Kuwana, H. Kameda and T. Takeuchi, "Sensitivity and Specificity of 2010 Rheumatoid Arthritis Classification Criteria," Rheumatology, Vol. 50, No. 7, 2011, pp. 1268-1274. doi:10.1093/rheumatology/keq442

[6] A. F. Mourão, et al., "Markers of Progression to Rheumatoid Arthritis: Discriminative Value of the New ACR/ EULAR Rheumatoid Arthritis Criteria in a Portuguese Population with Early Polyarthritis," Acta Reumatologica Portuguesa, Vol. 36, No. 4, 2011, pp. 370-376.
[7] H. Kokkonen, et al., "Antibodies of IgG, IgA and IgM Isotypes against Cyclic Citrullinated Peptide Precede the Development of Rheumatoid Arthritis," Arthritis Research \& Therapy, Vol. 13, No. 1, 2011, p. R13. doi:10.1186/ar3237

[8] D. Aletaha, F. Alasti and J. S. Smolen, "Rheumatoid Factor Determines Structural Progression of Rheumatoid Arthritis Dependent and Independent of Disease Activity," Annals of Rheumatic Diseases, 2012.

[9] C. Alessandri, R. Priori, M. Modesti, R. Mancini and G. Valesini, "The Role of Anti-Cyclic Cytrullinate Antibodies Testing in Rheumatoid Arthritis," Clinic Reviews in Allergy \& Immunology, Vol. 34, No. 1, 2008, pp. 45-49. doi:10.1007/s12016-007-8023-4

[10] L. A. van de Stadt, M. H. de Koning, R. J. van de Stadt, G. Wolbink, B. A. Dijkmans, et al., "Development of the Anti-Citrullinated Protein Antibody Repertoire Prior to the Onset of Rheumatoid Arthritis," Arthritis \& Rheumatism, Vol. 63, No. 11, 2011, pp. 3226-3233. doi:10.1002/art.30537

[11] V. P. Bykerk and J. M. Hazes, "When Does Rheumatoid Arthritis Start and Can It Be Stopped before It Does?" Annals of the Rheumatic Diseases, Vol. 69, No. 3, 2010, pp. 473-475. doi:10.1136/ard.2009.116020

[12] J. Nam, B. Combe and P. Emery, "Early Arthiritis: Diagnosis and Management," Eular On-Line Course on Rheumatic Diseases, Module 13-2007-2009 EULAR.

[13] S. Bosello, A. L. Fedele, G. Peluso, E. Gremese, B. Tolusso and G. Ferraccioli, "Very Early Rheumatoid Arthritis Is the Major Predictor of Major Outcomes: Clinical ACR Remissionand Radiographic Non-Progression," Annals of the Rheumatic Diseases, Vol. 70, No. 7, 2011, pp. 1292-1295. doi:10.1136/ard.2010.142729

[14] P. Emerty, "Evidence Supporting the Benefit of Early Interventionin Rheumatoid Arthritis," Journal of Rheumatology, Vol. 66, 2002, pp. 3-8.

[15] P. G. Conaghan, "Predicting Outcomes in Rheumatoid Arthritis," Clinical Rheumatology, Vol. 30, No. 1, 2011, pp. 41-47. doi:10.1007/s10067-010-1639-4

[16] H. Visser, S. le Cessie, K. Vos, F. C. Breedveld and J. M. Hazes, "How to Diagnose Rheumatoid Arthritis Early: A Prediction Model for Persistent (Erosive) Arthritis," Arthritis \& Rheumatism, Vol. 46, No. 2, 2002, pp. 357-365. doi:10.1002/art.10117

[17] M. Schoels, C. Bombardier and D. J. Aletaha, "Diagnostic and Prognostic Value of Antibodies and Soluble Biomarkers in Undifferentiated Peripheral Inflammatory Arthritis: A Systematic Review," The Journal of Rheumatology, Vol. 87, 2011, pp. 20-25. doi:10.3899/jrheum. 101070

[18] J. Anderson, L. Caplan, J. Yazdany, M. L. Robbins, T. Neogi, K. Michaud, K. G. Saag, J. R. O’Dell and S. Kazi, "Rheumatoid Arthritis Disease Activity Measures: American College of Rheumatology Recommendations for Use in Clinical Practice," Arthritis Care \& Research, Vol. 64, No. 5, 2012, pp. 640-647. doi:10.1002/acr.21649

[19] C. Gaujoux-Viala, G. Mouterde, A. Baillet, P. Claudepierre, B. Fautrel, X. Le Loët and J. F. Maillefert, "Evaluating Disease Activity in Rheumatoid Arthritis: Which 
Composite Index Is Best? A Systematic Literature Analysis of Studies Comparing the Psychometric Properties of the DAS, DAS28, SDAI and CDAI," Joint Bone Spine, Vol. 79, No. 2, 2012, pp. 149-155. doi:10.1016/i.jbspin.2011.04.008

[20] M. van den Broek, L. Dirven, J. K. de Vries-Bouwstra, A. J. Dehpoor, Y. P. Goekoop-Ruiterman, A. H. Gerards, P. J. Kerstens, T. W. Huizinga, W. Lems and C. F. Allaart, "Rapid Radiological Progression in the First Year of Early Rheumatoid Arthritis Is Predictive of Disability and Joint Damage Progression during 8 Years of Follow-Up," Annals of the Rheumatic Diseases, Vol. 71, No. 9, 2012, pp. 1530-1533. doi:10.1136/annrheumdis-2011-201212

[21] N. Vastesaeger, S. Xu, D. Aletaha, et al., "A Pilot Risk Model for the Prediction of Rapid Radiographic Progression in Rheumatoid Arthritis," Rheumatology, Vol. 48, No. 9, 2009, pp. 1114-1121. doi:10.1093/rheumatology/kep155

[22] K. Visser, Y. P. Goekoop-Ruiterman, J. K. de VriesBouwstra, et al., "A Matrix Risk Model for the Prediction of Rapid Radiographic Progression in Patients with Rheumatoid Arthritis Receiving Different Dynamic Treatment Strategies: Post Hoc Analyses from the BeSt Study," Annals of the Rheumatic Diseases, Vol. 69, No. 7, 2010, pp. 1333-1337. doi:10.1136/ard.2009.121160

[23] D. McGonagle, et al., "The Relationship between Synovitis and Bone Changes in Early Untreated Rheumatoid Arthritis: A Controlled Magnetic Resonance Imaging Study," Arthritis \& Rheumatism, Vol. 42, No. 8, 1999, pp. 1706-1711.

doi:10.1002/1529-0131(199908)42:8<1706::AID-ANR20> 3.0.CO;2-Z

[24] E. Olech, J. V. Crues III, D. E. Yocum and J. T. Merrill, "Bone Marrow Edema Is the Most Specific Finding for Rheumatoid Arthritis (RA) on Noncontrast Magnetic Resonance Imaging of the Hands and Wrists: A Comparison of Patients with RA and Healthy Controls," Journal of Rheumatology, Vol. 37, No. 2, 2010, pp. 265-274. doi:10.3899/jrheum.090062

[25] A. Filer, et al., "Utility of Ultrasound Joint Counts in the
Prediction of Rheumatoid Arthritis in Patients with Very Early Synovitis," Annals of the Rheumatic Diseases, Vol. 70, No. 3, 2011, pp. 500-507.

[26] S. Y. Kawashiri, T. Suzuki, A. Okada, S. Yamasaki, M. Tamai, H. Nakamura, T. Origuchi, A. Mizokami, M. Uetani, K. Aoyagi, K. Eguchi and A. Kawakami, "Musculoskeletal Ultrasonography Assists the Diagnostic Performance of the 2010 Classification Criteria for Rheumatoid Arthritis," Modern Rheumatology, 2012. doi:10.1007/s10165-012-0628-7

[27] G. Labanauskaite and V. Sarauskas, "Correlation of Power Doppler Sonography with Vascularity of the Synovial Tissue," Medicina, Vol. 39, No. 5, 2003, pp. 480483.

[28] R. J. Wakefield, M. J. Green, H. Marzo-Ortega, P. G. Conaghan, W. W. Gibbon, D. McGonagle, S. Proudman and P. Emery, "Should Oligoarthritis Be Reclassified? Ultrasound Reveals a High Prevalence of Subclinical Disease," Annals of the Rheumatic Diseases, Vol. 63, No. 4, 2004, pp. 382-385. doi:10.1136/ard.2003.007062

[29] M. Green, H. Marzo-Ortega, D. McGonagle, R. Wakefield, S. Proudman, P. Conaghan, J. Gooi and P. Emery, "Persistence of Mild, Early Inflammatory Arthritis: The Importance of Disease Duration, Rheumatoid Factor, and the Shared Epitope," Arthritis \& Rheumatism, Vol. 42, No. 10, 1999, pp. 2184-2188. doi:10.1002/1529-0131(199910)42:10<2184::AID-ANR2 0>3.0.CO;2-2

[30] V. P. Nell, K. P. Machold, G. Eberl, T. A. Stamm, M. Uffmann and J. S. Smolen, "Benefit of Very Early Referral and Very Early Therapy with Disease-Modifying Anti-Rheumatic Drugs in Patients with Early Rheumatoid Arthritis," Rheumatology, Vol. 43, No. 7, 2004, pp. 906-914. doi:10.1093/rheumatology/keh199

[31] A. Finckh, M. H. Liang, C. M. van Herckenrode and P. de Pablo, "Long-Term Impact of Early Treatment on Radiographic Progression in Rheumatoid Arthritis: A MetaAnalysis," Arthritis Care \& Research, Vol. 55, No. 6, 2006, pp. 864-872. doi:10.1002/art.22353 